\title{
Construction pas à pas d'une liaison par fibre optique longue distance
}

\author{
Eric Cassan ${ }^{1}$, Stéphanie Cassan ${ }^{2}$ \\ 1 : Faculté des Sciences d’Orsay, Bât 220, Université Paris-Sud 11 Orsay, 91405 Orsay Cedex, \\ eric.cassan@ief.u-psud.fr \\ 2 : ENS de Cachan, 61 avenue du président Wilson, 94235 Cachan Cedex, stephanie.cassan@eea.ens-cachan.fr
}

RESUME Cet article présente une expérience menée en partenariat entre la Faculté des Sciences d'Orsay et l'ENS de Cachan pour l'enseignement des télécommunications optiques au sein du Master IST (Information, Systèmes, Technologie, ex-EEA). Un logiciel commercial (COMSIS), permettant une description fonctionnelle des chaînes de communication par fibres optiques, est utilisé au cours de deux séances de TP sur cette thématique. Cette approche permet une étude globale des liaisons simples ou multiplexées en longueur d'onde (WDM), en mettant l'accent sur les principales limitations introduites par les différents composants optiques (fibre, source laser, amplificateur optique,...). L'utilisation du logiciel permet donc de construire progressivement une liaison par fibre optique longue distance ( $>300$ $\mathrm{km})$, les différents éléments de la liaison étant étudiés successivement et l'accent étant mis sur les mécanismes à compenser afin de garantir un taux d'erreur par bit donné (typiquement $10^{-15}$ ) à un débit binaire de quelques Gbits $\times \mathrm{s}^{-1}$. Cet article s'attache à montrer qu'en dépit des qualités extraordinaires de la fibre optique, la construction d'une liaison longue distance nécessite la compensation des phénomènes d'atténuation et de dispersion chromatique, ainsi que des effets optiques non-linéaires, message principal que l'on cherche à faire passer auprès des étudiants.

Mots clés : Télécommunications optiques, fibre optique, atténuation, dispersion chromatique, effets optiques nonlinéaires, émetteurs, amplificateurs EDFA, taux d'erreur par bit, diagramme de l'œil.

\section{1 . CONTEXTE ET OBJECTIFS}

\subsection{Bref historique}

Les télécommunications par fibres optiques, dont le développement a sensiblement débuté il y a 30 ans, ont connu plusieurs « révolutions » [1-4].

La première étape a été marquée par l'invention du laser au début des années soixante, puis la mise au point de fibres optiques à très faibles pertes vers 1970 . Les années soixante dix ont vu ensuite la réalisation de diodes laser performantes (diodes GaAs, puis InP). Ces avancées ont permis la conception puis la réalisation des premières liaisons point à point mono-longueur d'onde. Une étape supplémentaire a été franchie avec l'invention en 1989 de l'amplificateur à fibre dopée Erbium ou EDFA (voir Figure 1).

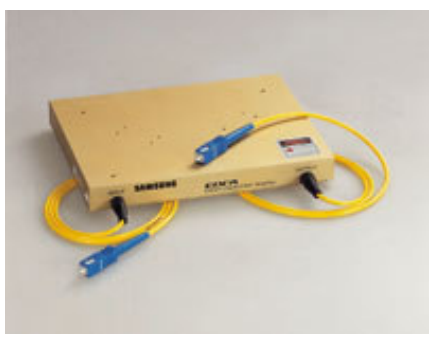

fig. 1 : photographie d'un amplificateur EDFA

\subsection{Conception d'une liaison moyenne distance}

La Figure 2 présente les pertes de propagation de la fibre optique silice en fonction de la longueur d'onde. Ces pertes sont minimales dans le proche infra-rouge, vers $\lambda=1550 \mathrm{~nm}(0,2 \mathrm{~dB} / \mathrm{km} \leftrightarrow$ atténuation d'un facteur 100 après $100 \mathrm{~km}$ de propagation). Ces pertes peuvent être compensées par une amplification

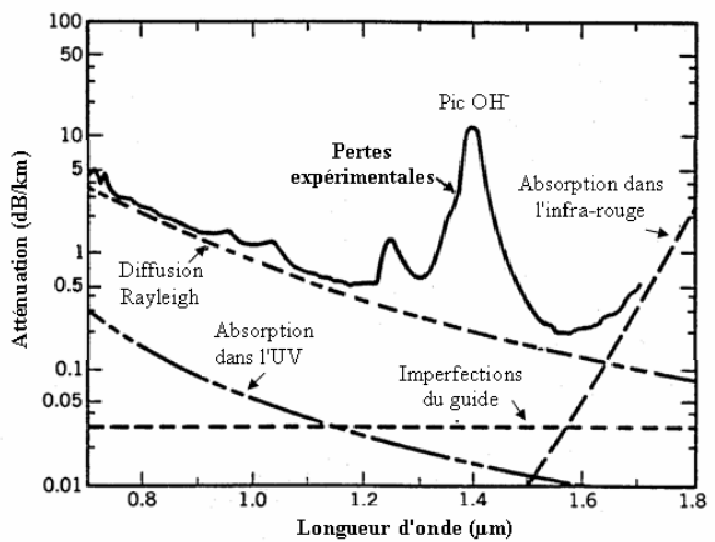

fig. 2 : pertes de propagation de la fibre optique

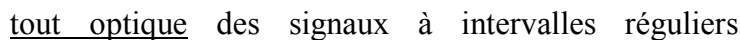
(typiquement tous les 50 à $100 \mathrm{~km}$ ). Une fois les pertes de propagation bien compensées, un autre problème apparaît pour l'établissement de liaisons longue distance: la dispersion chromatique de la fibre, provenant de la variation de la vitesse de groupe de la lumière avec la longueur d'onde [5]. Les sources optiques n'étant pas monochromatiques, il en résulte en effet un étalement temporel des impulsions optiques véhiculées, ce qui limite le débit binaire des liaisons. Des techniques particulières de compensation de la dispersion chromatique ont donc été mises en place : fibre à dispersion décalée, ingénierie du profil d'indice de la fibre, insertion de fibres à dispersion négative, réseaux de Bragg compensateurs, etc ... [3,4].

Ces diverses améliorations ont permis d'accéder à des débits importants $\left(>>\right.$ Gbits $\left.\times \mathrm{s}^{-1}\right)$ sur des distances assez longues $(>200-300 \mathrm{~km})$. 


\subsection{Un pas de plus : le multiplexage en longueur d'onde}

Devant l'augmentation de la densité du réseau optique (voir Figure 3) et des flux d'informations nécessaires, notamment $\mathrm{du}$ fait du développement d'Internet, l'utilisation d'une unique porteuse optique vers $1550 \mathrm{~nm}$ est apparue comme extrêmement contraignante.

Formulée plusieurs années auparavant, la technique du multiplexage en longueur d'onde (en anglais Wavelength Division Multiplexing, ou WDM) a débouché au plan industriel en 1997 et a rapidement envahi une très large partie des réseaux [3]. Compte tenu de la large plage de longueurs d'ondes autour de $1550 \mathrm{~nm}$ où la fibre présente de faibles pertes, on utilise non pas une mais tout un ensemble de porteuses optiques très proches (espacées de quelques $0,1 \mathrm{~nm}$ ), éventuellement des niveaux de puissance importants en entrée $(>>1 \mathrm{~mW})$ dans une fibre dont le rayon de cœur n'est que de l'ordre de $5 \mu \mathrm{m}$.

\subsection{Vers une liaison longue distance}

Lorsque la liaison ne dépasse pas la centaine de kilomètres et que le niveau de puissance optique injecté reste inférieur à quelques $\mathrm{mW}$, le multiplexage en longueur d'onde permet d'obtenir des débits « agglomérés » très élevés. Dès lors que l'on cherche à augmenter davantage la distance et/ou la puissance injectée en début de liaison, se manifestent dans la silice - matériau pourtant très linéaire - des effets optiques non-linéaires à l'origine de diverses dégradations telles que le "mélange à 4 ondes ». Ce phénomène se traduit par un échange d'énergie tout à fait involontaire entre des canaux en longueurs d'onde normalement isolés [4].

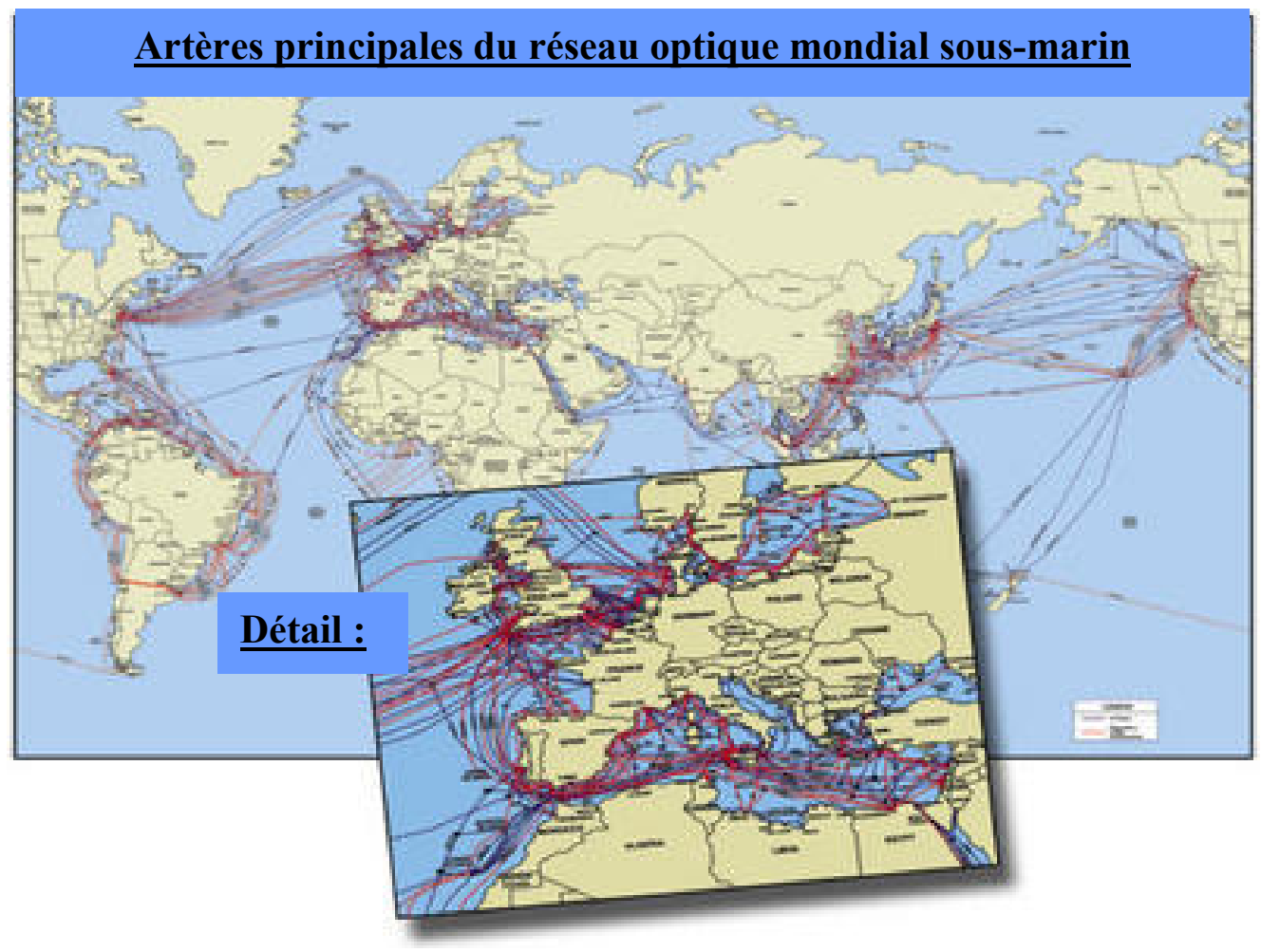

fig. 3 : principales voies fibrées sous-marines du monde - $\underline{\text { A noter }}$ : le très dense réseau terrestre mondial ne figure pas

chacune étant modulée en amplitude à un débit de quelques Gbits $\times \mathrm{s}^{-1}$, l'ensemble constituant un peigne de canaux en longueurs d'ondes ou canaux WDM. Du fait de la linéarité des équations de Maxwell et des caractéristiques linéaires de la silice, ces différentes longueurs d'ondes ou «couleurs » n'interagissent pas entre elles, mais se propagent quasiment de manière indépendante.

Les pertes de propagation et la dispersion chromatique étant bien compensées, de longues distances de propagation $(>>100 \mathrm{~km})$ sont envisageables, avec
On parle alors de diaphotie entre canaux.

Ces effets, masqués en présence de dispersion chromatique, n'apparaissent que lorsqu'elle est compensée et sont même d'autant plus forts que la dispersion est mieux compensée [3] ! L'élimination des effets non-linéaires nécessite donc une gestion particulière de la dispersion chromatique (« dispersion management $»)$ : on préfère tolérer une légère dispersion chromatique, quitte à alterner des portions de fibre à dispersion légèrement positive et des portions de fibre à dispersion légèrement négative. 


\subsection{Objectifs du TP}

L'objectif poursuivi lors des deux séances de travaux pratiques est de faire comprendre aux étudiants cette gradation dans les mécanismes de compensation nécessaires à l'établissement d'une liaison longue distance. Cela demande de leur part une bonne connaissance des différents mécanismes et éléments des liaisons (émetteurs, amplificateurs optiques, détecteurs, etc ...). Ces séances de TP sont donc l'occasion de fréquents retours sur des points du cours. L'outil numérique utilisé (COMSIS : [6]) permet d'appréhender l'ensemble des phénomènes séparément et de manière très souple, sans approximation, et sans calculs analytiques (tout à fait impraticables compte tenu des liaisons mises en oeuvre) [7]. Le TP est également l'occasion de revenir sur des notions et des outils très généraux concernant les liaisons numériques (taux d'erreur, diagramme de l'œil, facteur $\mathrm{Q}, \ldots$ ), notions pas toujours bien maîtrisées par les étudiants.

\section{2 . LIAISON POINT A POINT} MONOLONGUEUR D'ONDE

La Figure 4 montre un exemple de liaison par fibre optique. une telle diode en modulation directe $?, \ldots$. On note en particulier que le débit binaire est limité par les oscillations de relaxation caractéristiques des diodes laser en régime de modulation (à quelques Gbits $\times \mathrm{s}^{-1}$ ).

\subsection{Etape 2 : compensation des pertes optiques de propagation}

Les conditions de bon fonctionnement de l'amplificateur EDFA sont ensuite évaluées, la fibre optique ayant une atténuation de $0,2 \mathrm{~dB} / \mathrm{km}$. On constate que la longueur de FIB1 doit être suffisamment grande pour éviter la saturation de l'EDFA, mais suffisamment courte pour éviter de noyer complètement le signal à amplifier dans le bruit propre lié à l'émission spontanée de lumière dans l'EDFA (ASE). Les étudiants ont majoritairement du mal à appréhender ces diverses notions, et un bref retour sur le cours est souvent nécessaire à ce stade. La longueur de FIB1 est finalement fixée à $120 \mathrm{~km}$.

L'amplification de l'EDFA étant de l'ordre de $30 \mathrm{~dB}$ dans ces conditions, le niveau de puissance optique en sortie $\{\mathrm{sa}\}$ est très élevé (quelques $\mathrm{mW}$ ).

\subsection{Analyse de la liaison}

Une fois que les pertes de propagation sont

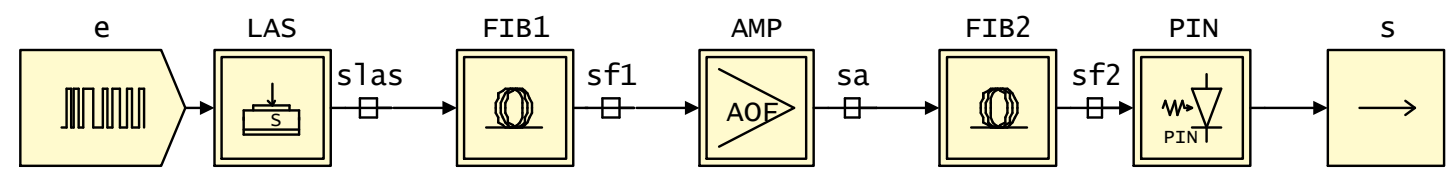

fig. 4 : Liaison point à point mono-longueur d'onde étudiée

\subsection{Description de la liaison}

En amont, un générateur de bits permet de commander une diode laser par une succession d'impulsions de courant, dont on règle les niveaux haut et bas, ainsi que le débit binaire. La diode laser, dont les caractéristiques sont choisies (notamment $\lambda=1,55 \mu \mathrm{m}$ ), émet dans une fibre optique monomode (notée FIB1). Afin de simplifier au maximum la liaison, on se ramène à une situation à un seul amplificateur optique (noté AMP), qui permet d'augmenter la distance totale de la liaison, constituée des longueurs additionnées des sections des fibres FIB1 et FIB2.

\subsection{Etape 1 : réglage de la source laser}

L'analyse de la liaison débute par l'observation des caractéristiques de la source. On demande d'observer et de commenter la forme des impulsions optiques émises par la diode laser en fonction du débit binaire qu'on lui

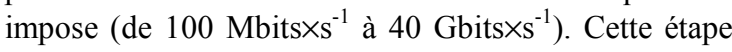
permet d'évaluer les acquis des étudiants concernant la compréhension des conditions de fonctionnement des diodes laser : comment choisir les niveaux haut et bas en courant sur la caractéristique statique puissancecourant de la diode ?, jusqu'à quel débit peut-on utiliser compensées, on demande alors aux étudiants d'évaluer le taux d'erreur par bit (TEB) de la liaison après seulement $10 \mathrm{~km}$ de propagation dans la fibre FIB2. Cette estimation passe par l'affichage du diagramme de l'œil de la liaison $(\mathrm{e} \rightarrow \mathrm{s})$, dont l'ouverture permet une évaluation du TEB. Le résultat obtenu est surprenant : le TEB de la liaison est d'environ $5 \times 10^{-6}$, soit 5000 erreurs par seconde (!) si le débit binaire est de 1 Gbits $\times \mathrm{s}^{-1}$, et ce en dépit du très fort niveau de puissance disponible au niveau du détecteur (noté PIN). L'interprétation demandée aux étudiants permet d'identifier la cause de ce résultat décevant: l'atténuation de la fibre est compensée par la présence de l'EDFA, mais pas la dispersion chromatique.

\subsection{Etape 3 : mise en évidence de l'effet de la dispersion chromatique - compensation}

Pour mettre en évidence l'effet de la dispersion chromatique, on annule le coefficient d'atténuation de la fibre optique et on analyse le signal $\{s f 1\}$. L'effet de la dispersion chromatique est alors étudié en fonction du débit binaire ( 1 à 5 Gbits $\times \mathrm{s}^{-1}$ ) et de la longueur de la liaison (200 à $1000 \mathrm{~km})$. Cette expérience permet d'observer l'effet de la dispersion chromatique de la 
fibre, dans le domaine temporel (étalement temporel) et dans le domaine spectral (distorsion du spectre vers les courtes ou grandes longueurs d'ondes en fonction du signe du coefficient de dispersion chromatique).

Une technique de compensation est ensuite mise en place, qui permet d'annuler le coefficient de dispersion chromatique de la fibre (cf section 1.2). Le TEB de la liaison est alors de nouveau évalué pour un débit binaire constant égal à $1 \mathrm{Gbits} \times \mathrm{s}^{-1}$, et ce en fonction de la longueur $\mathrm{L}_{2}$ de FIB2. Le résultat est reporté sur la Figure 5.

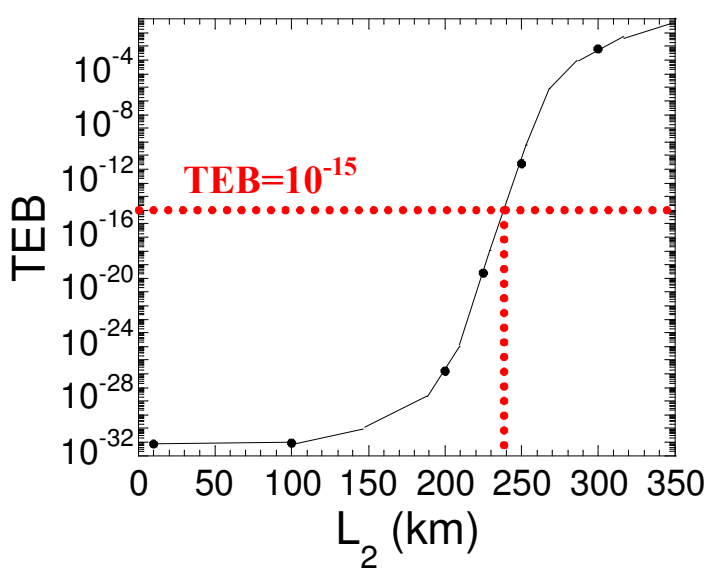

fig. 5 : évolution du TEB de la liaison en fonction de la longueur de la deuxième section de fibre (pour un débit binaire de 1 Gbits $\times s^{-1}$ )

On observe que la longueur maximale de la fibre optique FIB2 permettant de garantir un TEB de $10^{-15}$ est d'environ $240 \mathrm{~km}$. Cette distance est bien plus grande qu'en l'absence de correction de la dispersion chromatique. La longueur totale de la liaison est alors de $360 \mathrm{~km}$.

\section{3 . MELANGE A QUATRE ONDES}

L'influence des effets non-linéaires de la fibre optique est appréhendée dans une seconde liaison. Afin de mettre l'accent sur ce point, la liaison est simplifiée au maximum (voir Figure 6).
Cette liaison est constituée de deux sources optiques de longueurs d'onde proche: $\lambda_{1}=1550 \mathrm{~nm}$ et $\lambda_{2}=1551,6 \mathrm{~nm}$, émettant des impulsions gaussiennes. Ces deux longueurs d'onde sont injectées dans la même fibre optique au moyen d'un multiplexeur optique $2 \rightarrow 1$. On étudie alors le signal se propageant dans la fibre en fonction de la puissance des impulsions optiques injectées (en $\mathrm{dBm}$ ) et des caractéristiques de la fibre : longueur de la fibre, coefficient de dispersion chromatique, et coefficient Kerr $\left(\mathrm{k} \approx 3,2 \times 10^{-20} \mathrm{~m}^{2} / \mathrm{W}\right)$.

\subsection{L'effet Kerr}

L'effet Kerr correspond aux non-linéarités d'ordre 3 (terme en $\chi^{(3)}$ de la susceptibilité) [1,2]. Lorsqu'une onde se propage dans la fibre, elle entraîne alors une variation quasi instantanée de l'indice de réfraction de la silice proportionnelle à l'intensité optique $\mathrm{I}\left[\mathrm{W} / \mathrm{m}^{2}\right]$ :

$$
\mathrm{n}(\mathrm{t})=\mathrm{n}_{0}+\mathrm{k} \times \mathrm{I}(\mathrm{t})
$$

La surface équivalente du mode est définie par $\mathrm{P}=\mathrm{S} \mathrm{I}_{0}$ où $\mathrm{I}_{0}$ est l'intensité optique maximum de la distribution d'intensité qui est sensiblement gaussienne et $\mathrm{S}$ la «section» du mode optique guidé. Dans les fibres, la variation d'indice effectif par effet Kerr optique est obtenue en remplaçant $\mathrm{I}(\mathrm{t})$ par $\mathrm{P}(\mathrm{t}) / \mathrm{S}$. Bien que la variation d'indice puisse paraître négligeable $\left(\approx 10^{-10}\right)$, le déphasage non linéaire a des effets non négligeables compte tenu de la grande longueur de la fibre et de la très courte durée des impulsions généralement transmises.

Lors du TP, les effets non-linéaires sont appréhendés de manière très qualitative, sans le formalisme du tenseur liant la polarisation du milieu au champ électrique excitateur. Le mélange de fréquences et l'apparition de nouvelles fréquences optiques dans la fibre sont introduits par analogie avec des fonctions non-linéaires de l'électronique analogique, telles que les multiplieurs.

\subsection{Analyse de la liaison à puissance incidente constante $\left(P_{i}=1 \mathrm{~mW}\right)$}

Dans un premier temps, la longueur de la liaison est fixée à $50 \mathrm{~km}$, le coefficient de dispersion chromatique de la fibre est annulé, et aucun effet non-linéaire n'est pris en compte $(\mathrm{k}=0)$. On demande alors de procéder à

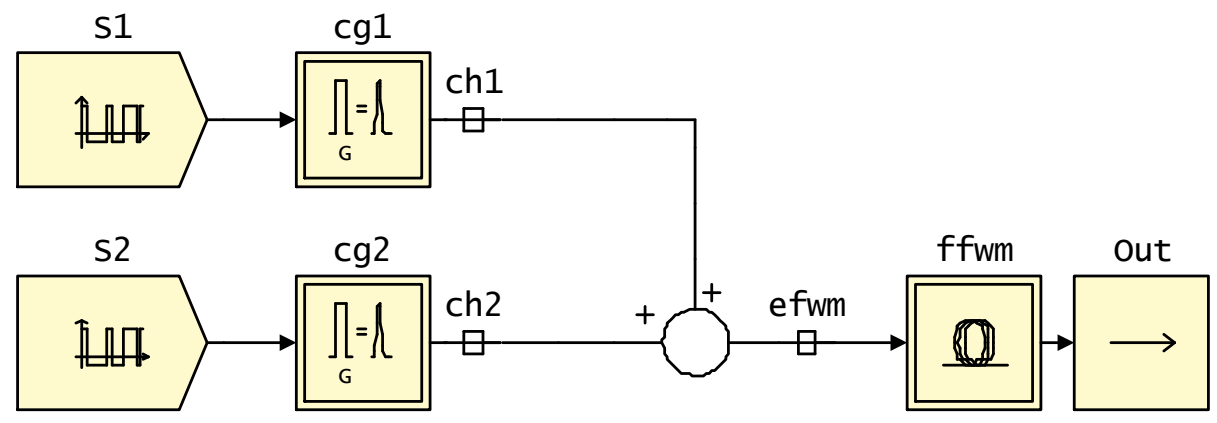

fig. 6 : Liaison étudiée pour illustrer l'influence du mélange à quatre ondes 
une première simulation temporelle de la liaison pour une puissance incidente de $0 \mathrm{dBm}(1 \mathrm{~mW})$.

Cette première manipulation permet d'observer l'absence de diaphotie entre les deux canaux centrés autour de $\lambda_{1}$ et de $\lambda_{2}$, ainsi que l'absence de tout canal parasite.

Dans un second temps, on demande aux étudiants d'introduire l'effet Kerr, la dispersion chromatique de la fibre étant toujours nulle.

Sont reportés sur la Figure 7 les densités spectrales de puissance normalisées $(\max =0)$ en entrée et en sortie de la section de fibre. On y observe clairement l'apparition de canaux parasites (mélange à 4 ondes). On insiste alors sur les problèmes engendrés par ce type de phénomènes dans les systèmes optiques multiplexés en longueurs d'ondes (WDM). sensiblement à la même vitesse. Or, cette condition est d'autant mieux vérifiée que la dispersion chromatique de la fibre est faible, puisque la dispersion chromatique a pour origine la variation de la vitesse de groupe de la lumière avec la longueur d'onde. La compensation de la dispersion chromatique aggrave donc les phénomènes de diaphotie dus aux effets non-linéaires ! Plutôt que d'annuler complètement la dispersion chromatique, on est donc amené à en tolérer un faible niveau (« dispersion management»), quitte à alterner les sections de fibre à dispersion positive et les sections de fibre à dispersion négative.

3.3 Influence de la puissance injectée dans la fibre La puissance du signal couplée en entrée joue également un rôle important dans l'apparition des effets

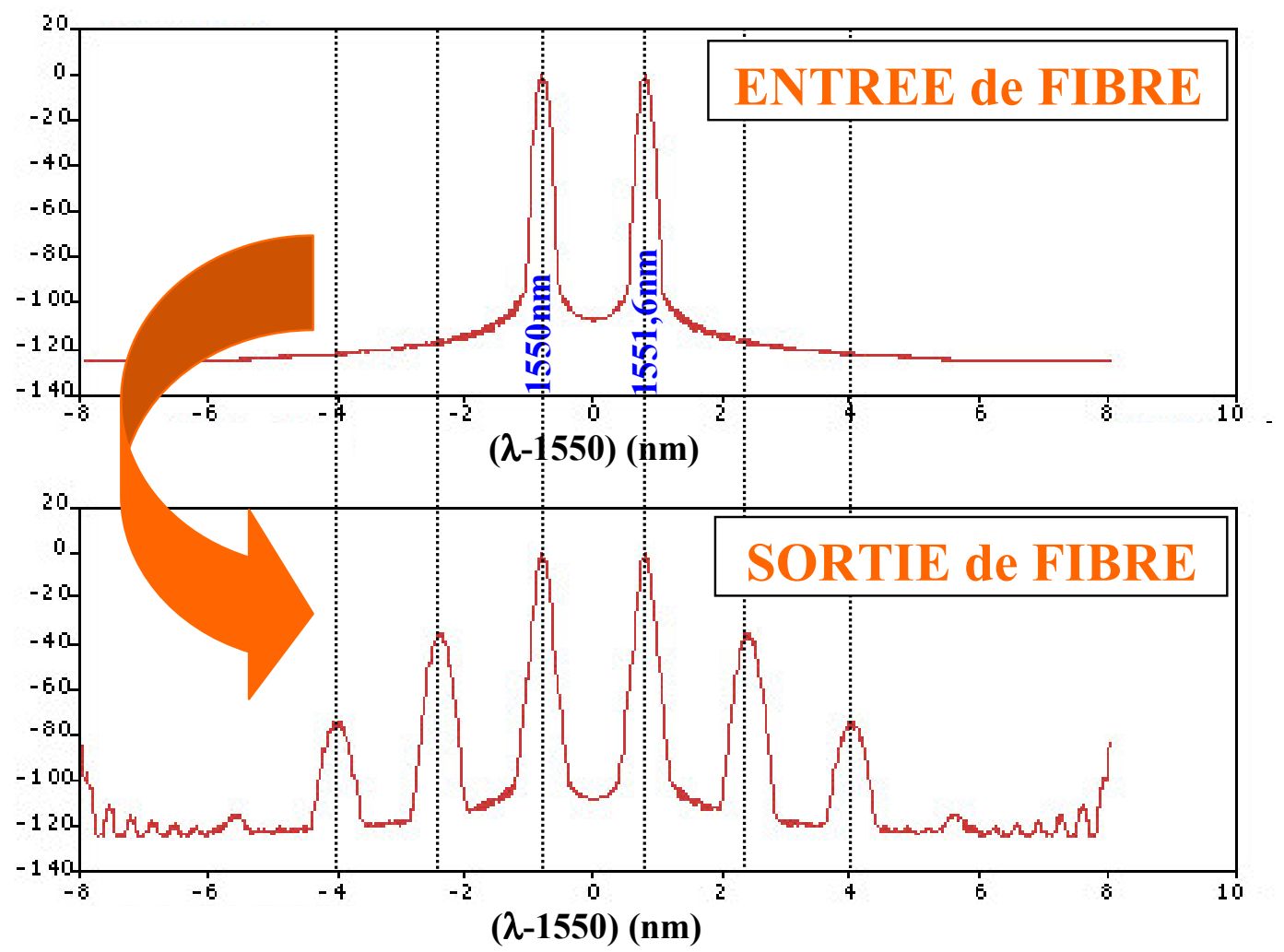

fig. 7 : Naissance de canaux parasites en longueur d'onde par « mélange à quatre ondes » dans la fibre

On essaie ensuite de réduire cet effet de diaphotie en étudiant l'influence de la dispersion chromatique. Le coefficient de dispersion chromatique de la fibre est modifié, passant de 0 à $2 \mathrm{ps} /(\mathrm{nm} \times \mathrm{km})$, puis à 17 $\mathrm{ps} /(\mathrm{nm} \times \mathrm{km})$. On constate alors que l'intensité du 'mélange à 4 ondes' diminue. Une interprétation qualitative est alors donnée aux étudiants. Pour se manifester, les effets non-linéaires nécessitent une condition d'accord de phase entre les ondes électromagnétiques se propageant dans la fibre. Deux ondes de longueurs d'ondes différentes ne peuvent en effet échanger de l'énergie que si elles se propagent non-linéaires. Les simulations mises en œuvre permettent de conclure que plus la puissance injectée en entrée est élevée, plus l'intensité des effets nonlinéaires est importante.

\section{CONCLUSION}

L'étude de ces deux liaisons, données ici à titre d'exemple, permet de tirer des conclusions sur la démarche à mettre en œuvre pour la réalisation de liaisons par fibres optiques longue distance: l'atténuation de la fibre doit être compensée périodiquement par des amplificateurs tout-optique 
(EDFA). La dispersion chromatique doit l'être également, ainsi que les effets optiques non-linéaires. Ces deux types de «pénalités» ne sont pas indépendantes, une trop bonne compensation de la dispersion tendant à renforcer les effets non-linéaires. La gestion de ces deux mécanismes doit donc être effectuée simultanément.

Un logiciel numérique qui décrit les chaînes de communications optiques est un outil intéressant, car il permet de séparer les différents phénomènes mis en jeu, et de modifier facilement des grandeurs telles que la longueur de liaison, le coefficient de dispersion chromatique, .... .

Deux séances de TP sont actuellement menées sur ce sujet dans le cadre du Master IST d'Orsay («Information, Systèmes, Technologie »). Ces séances s'apparentent à un cours-TP et semblent être bien ressenties par les étudiants.

\section{Bibliographie}

1.Zeno Toffano, Optoélectronique, Composants photoniques et fibres optiques, Ellipses (Technosup : Supelec), 2001.

2. Michel et Irène Joindot, Télécommunications par fibres optiques, Dunod,1996.

3.Harry J. R. Dutton, Understanding Optical Communications, presses IBM, première édition, 1998.

4. Govind P. Agrawal, "Fiber-Optic Communication Systems", WILEY, deuxième édition, 1997.

5.E Cassan, S. Cassan, Etude des propriétés physiques des fibres optiques à partir d'un modèle simple, Cetsis 2003, Toulouse.

6. COMSIS : http://www.ipsis.com.

7.E. Cassan, Une introduction aux télécommunications optiques par la simulation de systèmes simples, J3eA - Vol. 2 - 7 (2003). 